\title{
MADURACIÓN DEL FRUTO Y MORFOMETRÍA DE SEMILLAS DE Genipa americana L. EN EL DEPARTAMENTO DEL QUINDÍO
}

\author{
RIPENESS OF FRUIT AND SEED MORPHOMETRIC OF Genipa americana L. \\ IN THE DEPARTMENT OF THE QUINDIO
}

\author{
Sandra Viviana Ramírez Morales ${ }^{1}$ Andrés Felipe Orozco Cardona ${ }^{2}$
}

\author{
${ }_{1}$ Centro de Estudios e Investigaciones en Biodiversidad y Biotecnología (CIBUQ) \\ ${ }^{2}$ Programa de Biología, Investigador Centro de Estudios e Investigaciones en Biodiversidad y Biotecnología (CIBUQ), Universidad del Quindío. \\ Fecha de recibido: Febrero 3 de 2010 \\ Fecha de aceptado: Junio 9 de 2010 \\ Correspondencia: Centro de Estudios en Biodiversidad CIBUQ, Universidad del Quindío. Av. Bolivar calle 12 norte. Armenia Quindío. \\ Correo electrónico: andresorozco@uniquindio.edu.co
}

\section{RESUMEN}

Se evaluó el estado de maduración del fruto y la variabilidad morfométrica de las semillas de Genipa americana procedentes de 6 poblaciones de la zona cálida del departamento del Quindío. Se colectaron 60 frutos maduros de los cuales se tomaron 600 semillas para evaluar su morfometría. El estudio se desarrolló en el laboratorio de Biotecnología del Centro de Estudios e Investigaciones de Biodiversidad y Biotecnología de la Universidad del Quindío (CIBUQ). Se valoraron características organolépticas y morfométricas en los frutos; además de los caracteres: Peso, largo, ancho, grosor, relación largo-ancho, volumen y forma en las semillas, siguiendo la metodología de Iglesias, Mora y Casas (2005), con algunas modificaciones. Se calculó el coeficiente y factor de variación y se realizó un análisis de componentes principales mediante el programa Matlab (Versión 7.0). Se encontró que los frutos de la especie presentan variación en tamaño, peso y número de semillas; alto coeficiente y factor de variación en la forma de las semillas, varianza del 38\% en el primer componente principal y alta variabilidad intrapoblacional en volumen, peso y largo de las semillas. Los frutos y semillas de la especie presentan medidas superiores a lo reportado por otros autores. Es probable que factores genéticos afecten el tamaño peso y forma de la semilla; aunque la variación proviene principalmente de factores ambientales, que asimismo influencian la sobrevivencia, el crecimiento y la reproducción.

Palabras claves: Semillas forestales, madurez óptima, morfometría, Conservación.

\begin{abstract}
We evaluated the fruit ripeness state and the morphometric variability of Genipa americana seeds proceeding from 6 populations of the hot zone of the department of Quindio. There were collected 60 ripe fruits of which 600 seeds took to evaluate it's morfometry. The study was conducted in the Biotechnology laboratory of Study and Research on Biodiversity and Biotechnology Center at the University of Quindio (CIBUQ). We evalued the organoleptic and morfometric characteristics in the fruits; besides the characters: Weight, length, width, thickness, relation length - width, volume and seeds form, following the methodology of Mora and Casas (2005) with some modifications, to analyze population-level morphometric changes. We calculated thecoefficient and variation factor and we realized an principal components analysis by means of the program Matlab (Version 7.0). We found that G. americana fruits present variation in size, weight and number of seeds; equally a high coefficient and variation factor in the seeds form, variance of 38\% in the first principal component and high intrapopulation variability in size, weight and seeds lengthf. The specie fruits and seeds present higher measures to the reported. It is probable that genetic factors affect the size weight and seed forms; though the variation comes principally from environmental factors, which likewise influence the survival, the growth and the reproduction.
\end{abstract}

Key words: Forest seed, morphometry, Conservation 


\section{INTRODUCCIÓN}

Genipa americana, conocida comúnmente como Jagua, es una especie arbórea perteneciente a la familia Rubiaceae; crece naturalmente en ecosistemas húmedos tropicales de Colombia. Tiene gran valor alimenticio, medicinal, industrial y ecológico. Los indígenas usaban el tinte morado obtenido de los frutos inmaduros de la especie como pintura corporal (1-2). Además, ha sido utilizada como especie promisoria en modelos de recuperación de áreas degradadas en ambientes del Estado de São Paulo (3).

La especie exhibe un comportamiento no gregario, por lo cual se presentan individuos dispersos, rara vez más de dos por hectárea (4). A pesar de su amplia ocurrencia en zonas no preferenciales para la agricultura y protegidas por ley, la especie ha sufrido pérdida de variabilidad genética por la actividad humana predatoria e irresponsable (4). Algunos estudios previos realizados han permitido conocer que los individuos presentan una alta heterocigosidad, revelándola como una especie potencial para conservación in situ. Así mismo, la gran variabilidad genética evidenciada por la forma de los frutos, espesura de la pulpa y constituyentes químicos denota gran potencial para su uso en trabajos de mejoramiento de plantas (4).

Sin embargo, a pesar de la variación morfológica de sus semillas no se cuenta con suficientes estudios que aborden una caracterización morfométrica de las mismas. Por lo anterior, la presente investigación se desarrolló con el objetivo de evaluar la variación morfométrica en muestras de semillas que contribuyan en la elaboración de una estrategia de manejo y conservación de la especie.

\section{MATERIALES Y MÉTODOS}

\section{Área de estudio}

Se colectaron semillas de Genipa americana de 6 procedencias de la zona cálida del departamento del Quindío, en el Valle del río la Vieja (Figura 1).

Los sitios de muestreo correspondieron a: Reserva La Montaña del Ocaso, Vereda El Laurel y Vereda Puerto Alejandría, en el municipio de Quimbaya; Vereda Puerto Samaria y Vereda San Pablo, en el municipio de Montenegro; Vereda La Moravita en el municipio de Pijao y un último sitio localizado en la Vereda El

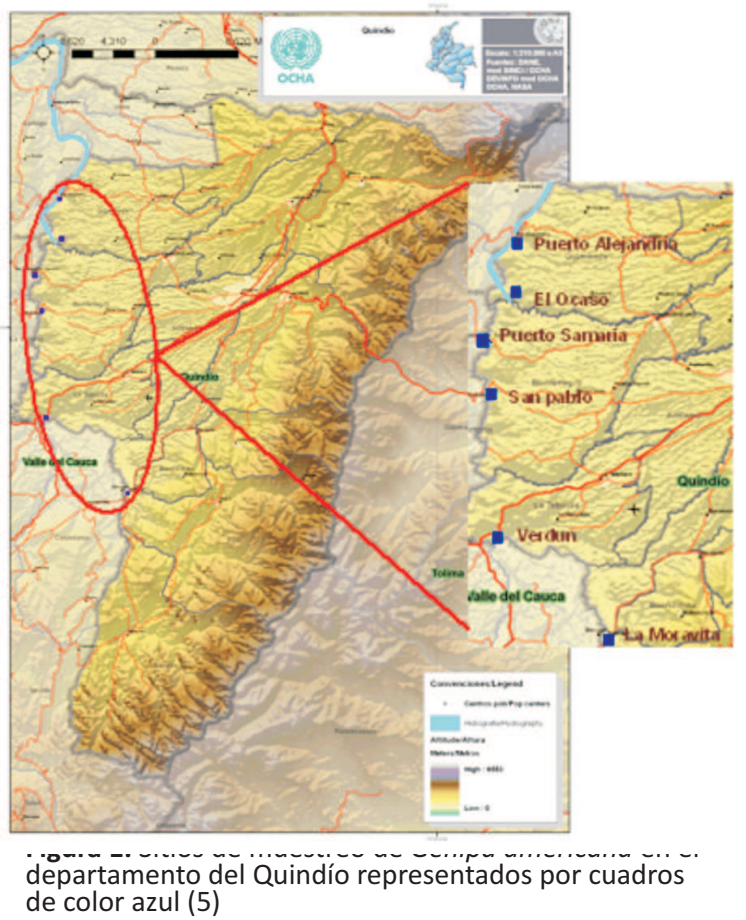

Verdún, municipio de Caicedonia, Valle del Cauca (Figura 1). La precipitación pluvial anual de la zona está entre los 1000-2000msnm y la temperatura media anual entre $18-24^{\circ} \mathrm{C}$.

El trabajo se llevó a cabo en dos fases:

\section{Fase de campo}

Se seleccionaron frutos maduros de árboles adultos con apariencia sana. De cada árbol se colectaron 10 frutos para cada una de las procedencias; los cuales posteriormente fueron trasladados al laboratorio de Biotecnología del Centro de Investigaciones en Biodiversidad y Biotecnología de la Universidad del Quindío (CIBUQ) para su evaluación.

\section{Fase de evaluación}

\section{- Estado de maduración del fruto}

Con el fin de identificar el estado de maduración óptimo del fruto y trabajar con semillas bien desarrolladas se hicieron mediciones de 60 frutos, 10 de cada sitio de muestreo, para la determinación del tamaño y peso adecuados (Figura 2). Los demás frutos colectados fueron descartados por tener tamaños muy pequeños o porque no llegaron a la madurez. Se tuvo en cuenta el ancho y largo, peso y número de semillas 
por fruto. Se utilizó un calibrador pie de Rey y una balanza digital Navigator Ohaus con $0,1 \mathrm{~g}$ de precisión. También se registraron características organolépticas como color, olor y consistencia de los frutos.

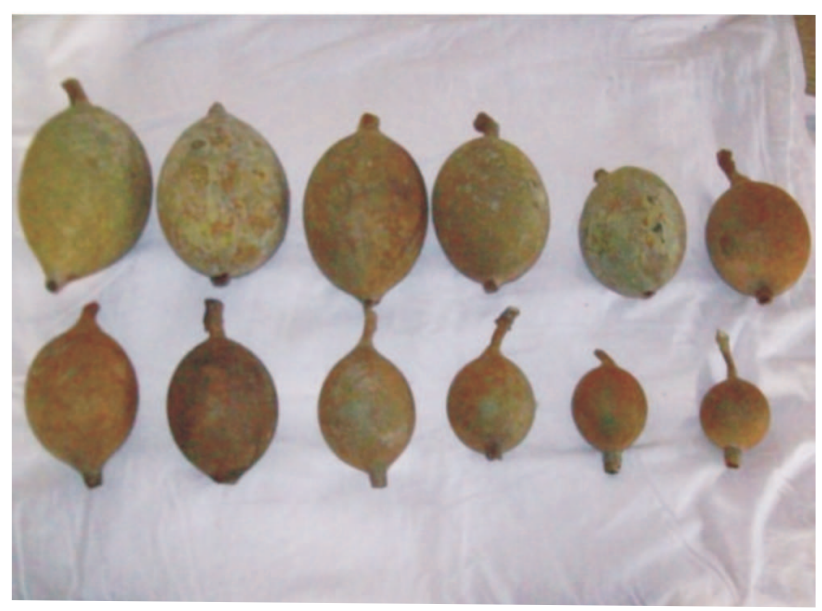

Figura 2. Estados de maduración del fruto de Genipa americana para el departamento Quindío.

\section{- Caracterización morfométrica}

El análisis morfométrico se realizó con el fin de establecer diferencias en las variables examinadas para todas las procedencias de las semillas.

La morfometría se realizó con los siguientes caracteres: Peso fresco de la semilla (PS), Largo (LS), ancho (AS), grosor (GS), relación largo ancho (L/AS), volumen (VS) y forma de la semilla (FS).

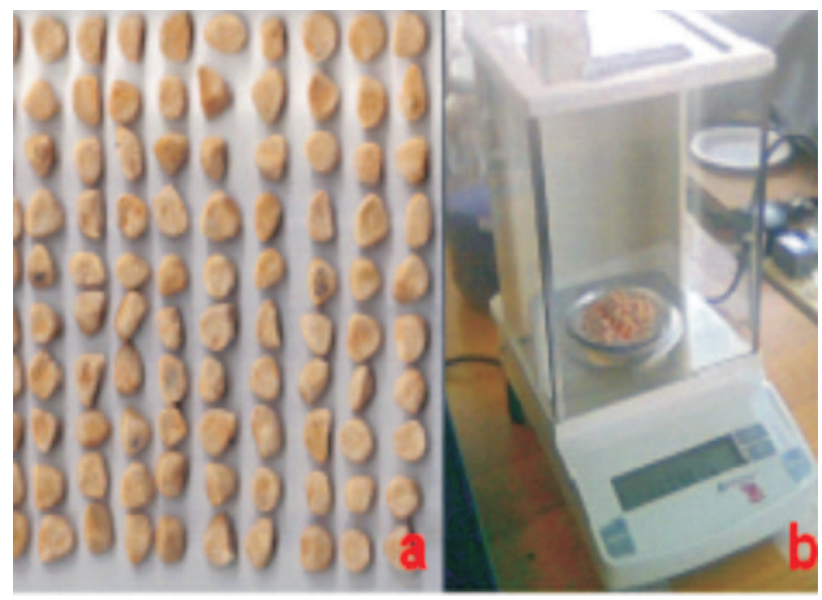

Figura 3. Toma de pesos de semillas de G. americana. Variables: a. Cantidad de semillas para pesaje. b. Balanza Analítica.
El peso de las semillas se determinó individualmente con una balanza digital de precisión 0,0001g y capacidad de 500g y se efectuó por grupos de 100 semillas (Figura 3).

Las medidas morfométricas tales como Largo (LS), Ancho (AS), Grosor (GS) y relación Largo / Ancho (L/ A) de la semilla (PS), se evaluaron de manera individual con un calibrador pie de Rey (Scale) con precisión de $0,1 \mathrm{~mm}$, en una muestra de 600 semillas.

La forma de la semilla fue determinada teniendo en cuenta 5 categorías, a saber: Triangular, categoría I; Circular, categoría II; Irregular, categoría III; Rectangular, categoría IV y Elipsoide, categoría

\section{Análisis estadístico}

Se elaboraron matrices de datos para cada variable evaluada. Se realizaron gráficas en Excel para la morfometría de los frutos. Se realizaron análisis de cada variable morfométrica evaluada en las semillas para describir las poblaciones, tales como: gráficas de estadística descriptiva, en los que se exhibieron datos como la media (Prom.), desviación estándar (Desv.), valor máximo (Máx.) y mínimo (Mín.), tanto para los datos sin tratamiento estadístico, como para los datos tratados estadísticamente. Se determinó el coeficiente de variación y factor de variación (6). Finalmente, se realizó un análisis de componentes principales mediante el programa MATLAB (Versión 7.0), en el cual se muestra la matriz de valores y vectores del análisis de los componentes de las variables que presentan en general mayor varianza en relación con las demás; se graficó la varianza explicada y acumulada de las componentes principales y para las componentes $1^{\circ} \mathrm{y}$ $2^{\circ}$ se mostró la distribución de los datos para cada procedencia en una gráfica bidimensional.

\section{RESULTADOS Y DISCUSIÓN}

\section{Estado de maduración del fruto}

A continuación se mencionan las características indicadoras del estado de madurez óptimo del fruto para la obtención de semillas que puedan ser utilizadas en almacenamiento:

Los frutos maduros de G. americana presentan un color café-parduzco en la totalidad del fruto y líquenes de color blanquecino en el exocarpo. Tienen una consistencia blanda al tacto, pero no se abren con facilidad. El olor es agrio y fuerte. Cuando el fruto está maduro carece de sustancias colorantes (Figura 4). 


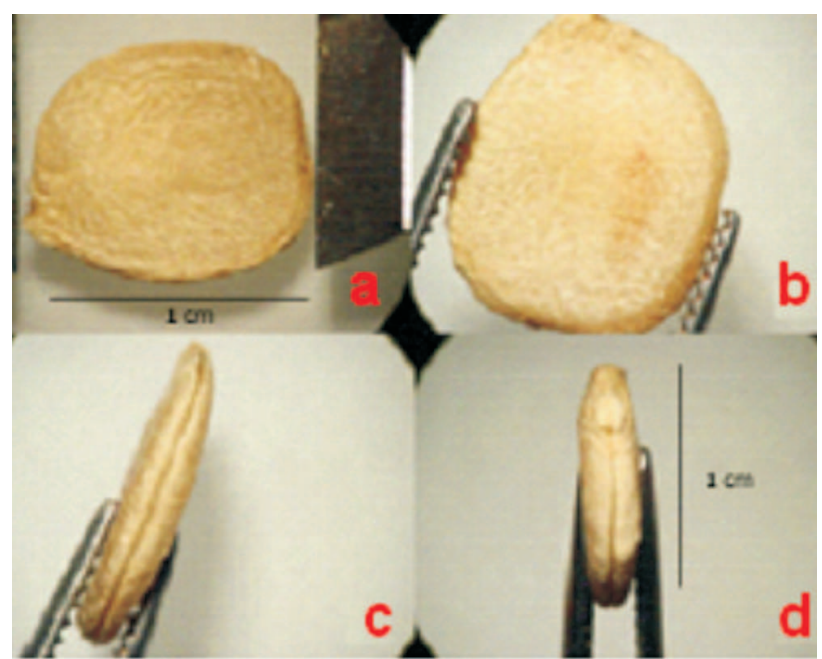

Figura 4. Estado óptimo de maduración de fruto y semillas de G. americana. a -b. Fruto maduro. c. Semillas recubiertas con fibras internas del mesocarpo.

En estado inmaduro el fruto posee un derivado de la genipina que al reaccionar con proteínas en presencia de oxígeno produce un pigmento azul (7).

El número de semillas por fruto fue 50-350; con un promedio de 170 semillas. Éstas se encuentran envueltas por fibras internas del mesocarpo, las cuales presentan color amarillo-blanquecino (Figura 4).

El tamaño óptimo de los frutos maduros para la colecta en el Quindío, de la especie G. americana es: Ancho 6,57-8,19 cm y largo 9,9-13,23 cm (Figura 5).

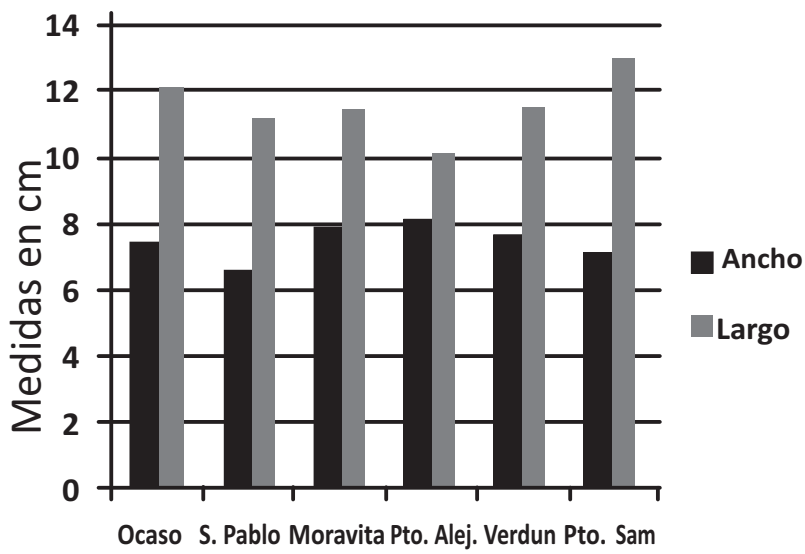

Figura 5. Morfometría del fruto de G. americana. Variable Tamaño: Ancho y largo del fruto de las seis procedencias.
El peso de los frutos de las seis procedencias varió entre 240-378 g (Figura 6).

\section{Peso de frutos de G. americana}

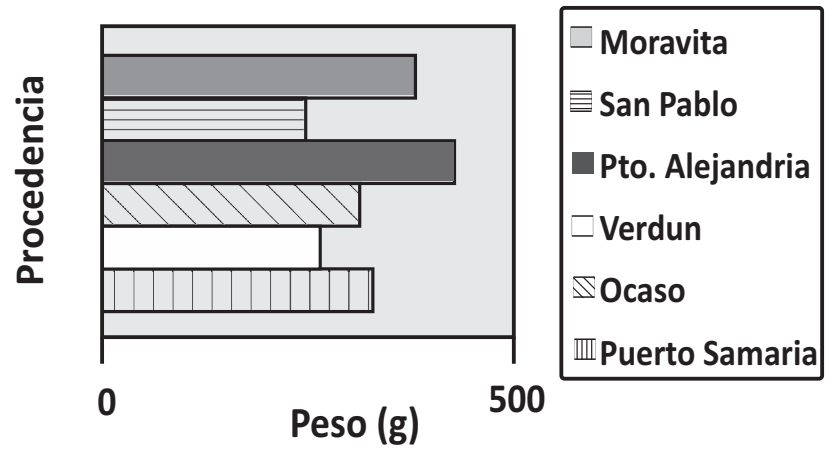

Figura 6. Morfometría del fruto de G. americana. Variable Peso para las seis Procedencias

Las características morfológicas correspondientes al estado de maduración del fruto son: pericarpo color marrón arrugado con semillas poco adheridas a la pulpa marrón amarillenta (8).

El tamaño, peso y número de semillas por fruto, presentó rangos de variación mayores a lo reportado en la literatura para la especie (4-9).

Algunos autores han indicado que los frutos maduros deben ser colectados cuando tienen el máximo de tamaño y se desprenden de la planta naturalmente (4).

G. americana tiene frutos dehiscentes, flotantes, cuya abscisión se produce en las temporadas de mayor índice de lluvias. Por lo tanto, el período de maduración coincide con el período de mayor precipitación, en el que la flotabilidad es reducida y ocurre un aumento de la densidad, peso y volumen de la fruta (10).

Adicionalmente, se ha mencionado que independientemente del grado de maduración del fruto de G. americana, existe incidencia fúngica en sus semillas. Probablemente, el grado de maduración morfológica de los frutos de G. americana no coincide con la maduración fisiológica de las semillas, conforme fue observado para semillas de otras especies (8).

\section{Análisis morfométrico}

En primer lugar la estadística descriptiva presenta un valor promedio del PS para las seis procedencias igual a 0,24 g, LS promedio 1,17 cm, AS promedio 0,92 cm, GS 
promedio $0,34 \mathrm{~cm}$, (Figuras 7 y $8 \mathrm{a}-\mathrm{b}$ ), L/AS promedio 1,23 vs promedio $0,38 \mathrm{~cm} 3$ y FS más común fue la categoría III, equivalente a semillas irregulares.

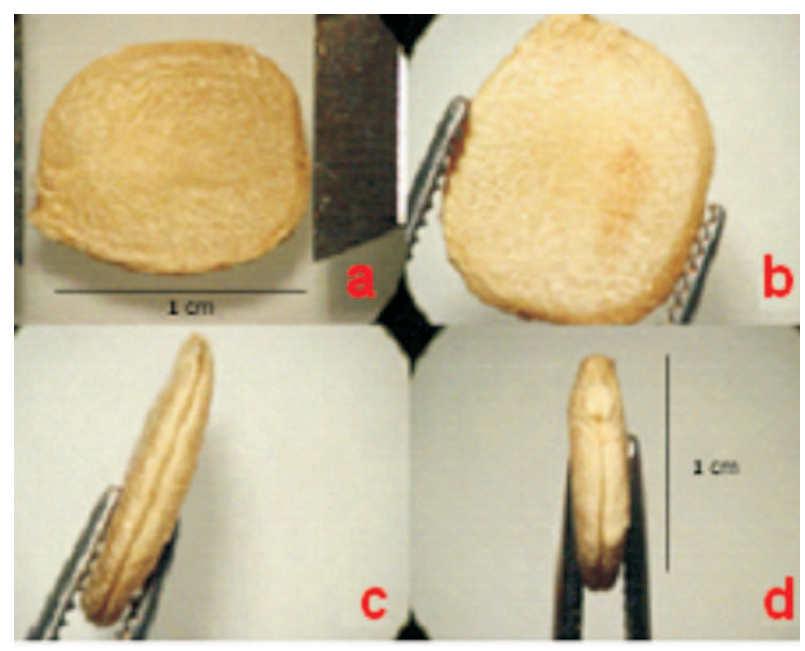

Figura 7. Medición de semillas de G. americana. Variables morfométricas: a. Largo, b. ancho, c. grosor. d. vista lateral semilla mostrando extremo micropilar.

Todos los sitios datos crudos
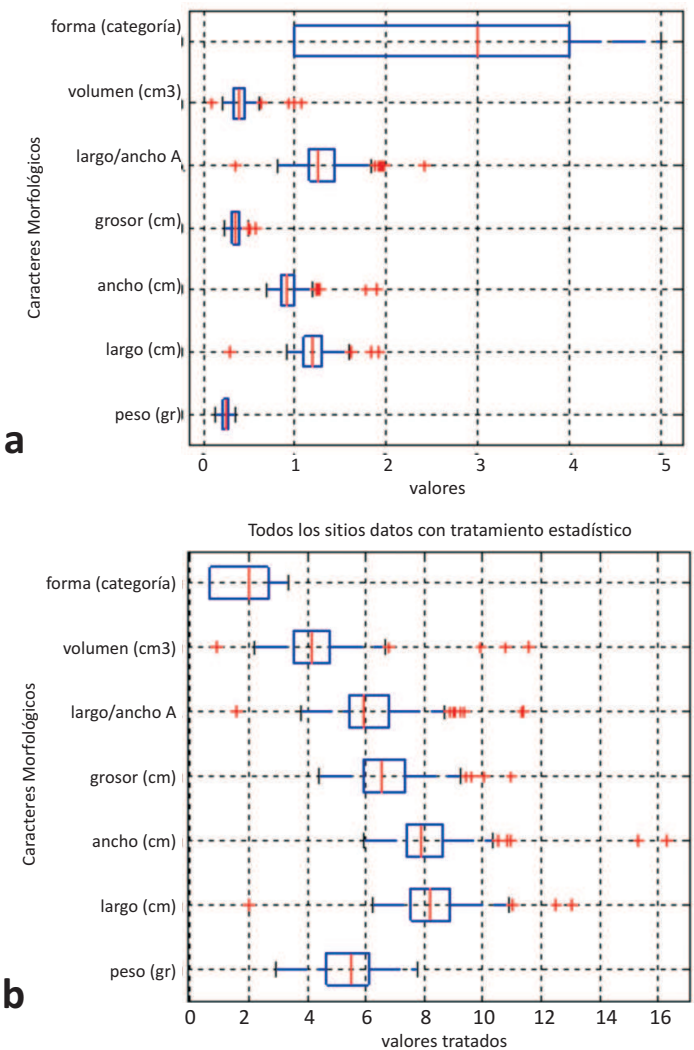

Figura 8. Datos de los caracteres morfométricos de semillas de G. americana. a. Sin tratamiento estadístico. b. Con tratamiento estadístico.

Rev. Invest. Univ. Quindío (21): 73- 81. Armenia - Colombia
Los resultados del análisis de los valores de coeficiente de variación y factor de variación obtenidos para las variables examinadas en las 6 procedencias (Figura 9) mostraron que la variable FS tuvo los valores más elevados para el coeficiente y factor de variación $(0,49$ y 2,23$)$.

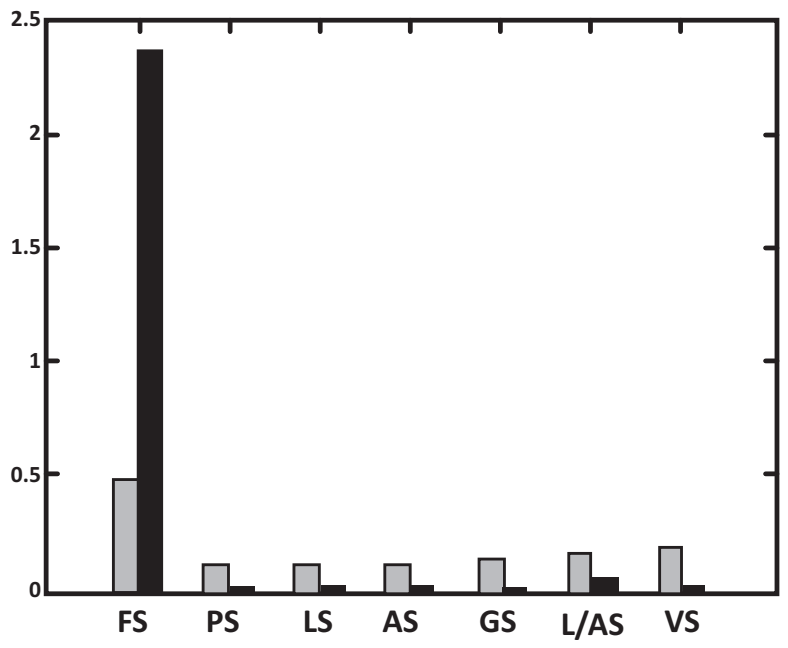

Figura 9. Variación en los valores de coeficiente y factor de variación para los caracteres morfométricos de la semilla evaluados: Forma (FS), peso (PS), largo (LS), ancho (AS), grosor (GS), relación largo/ancho (L/AS), volumen (VS).

Mientras que la variable LS presentó el valor más bajo en coeficiente de variación (0.08) y las variables GS, PS y VS tuvieron los menores valores en el factor de variación $(1,941 * 10-03 ; 6,46 * 10-03$ y 3,62*10-03 respectivamente) (Figura 9 ).

Lo anterior significa que las semillas presentan variación intrapoblacional en términos de la variable forma de las semillas; mientras que el largo, grosor, peso y volumen son características con pocas diferencias en las poblaciones evaluadas.

Los resultados del análisis de componentes principales (Tabla 1) revelaron que la componente 1 tiene la mayor correlación positiva con VS, PS y LS, mientras que tiene correlación negativa con FS.

La componente 2 tiene correlación positiva con L/AS y 
Tabla 1. Matriz de Valores y vectores del análisis de componentes principales para Las semillas de G. americana de seis procedencias del departamento de Quindío

\begin{tabular}{lccccc}
\hline \multirow{2}{*}{ Autovectores } & \multicolumn{5}{c}{ COMPONENTES PRINCIPALES } \\
\cline { 2 - 6 } PS & $\mathrm{C} 1$ & $\mathrm{C} 2$ & $\mathrm{C} 3$ & $\mathrm{C} 4$ & $\mathrm{C} 5$ \\
LS & 0,5035 & $-0,1352$ & 0,0096 & $-0,0691$ & $-0,8462$ \\
AS & 0,4631 & 0,3031 & 0,4582 & 0,1367 & 0,1597 \\
GS & 0,2853 & $-0,6058$ & 0,2633 & 0,0106 & 0,2591 \\
L/AS & 0,2997 & 0,1465 & $-0,7921$ & $-0,1793$ & 0,1197 \\
VS & 0,1627 & 0,6966 & 0,1527 & 0,0878 & 0,0165 \\
FS & 0,5772 & $-0,0932$ & $-0,1242$ & $-0,0431$ & 0,4178 \\
\hline
\end{tabular}

negativa con AS y PS. En la componente 3, se encontró correlación positiva con LS, GS y L/AS y negativa con FS. En la componente 4 se encontró correlación positiva con LS y L/AS y negativa con FS. La componente 5 tiene correlación positiva con LS, L/AS y AS y negativa para FS yGS.

Para las dos primeras componentes (Figura 10), se puede apreciar que la gran mayoría de observaciones se acumulan en los valores centrales de ambos componentes, aunque hay algunos datos que destacan por los valores distintos.

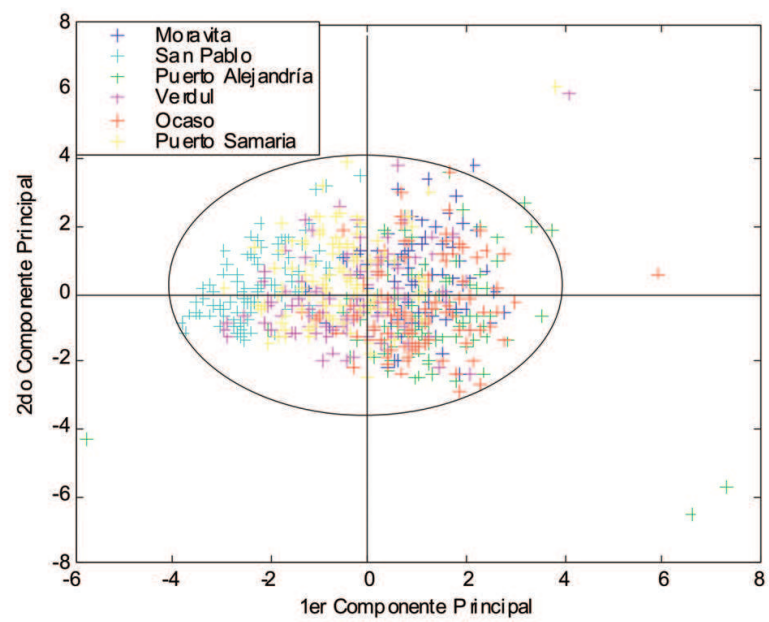

Figura 10. Gráfico bidimensional de las dos primeras componentes para las observaciones de semillas de G. americana de las seis procedencias.

Las semillas de la Moravita, El Ocaso y Puerto Alejandría presentan principalmente valores positivos para la primera componente, con algunos datos positivos y negativos en la segunda componente 0 datos atípicos.
Por el contrario, las semillas procedentes de San Pablo y Puerto Samaria muestran valores bajos para la primera componente principal y se distribuyen cerca al centro para la segunda componente.

En el caso de Verdun, las semillas se encuentran agrupadas cerca al centro y presentan un dato atípico.

En general las semillas del mismo municipio (San Pablo-Puerto Samaria y Ocaso-Puerto Alejandría) presentan mayor similaridad en variabilidad que entre los municipios (Figura 10).

La figura 11 exhibe la varianza explicada por cada uno de los cinco componentes principales que es de $37,88 \%$ para $C 1,26,25 \%$ para $C 2,15,47 \%$ para $C 3$, $14,11 \%$ para C4 y $5,77 \%$ para C5. La varianza acumulada es de $38 \%$ para $\mathrm{C} 1,64 \%$ para $\mathrm{C} 2,79 \%$ para C3, $93 \%$ para C4 y $99 \%$ para C5.

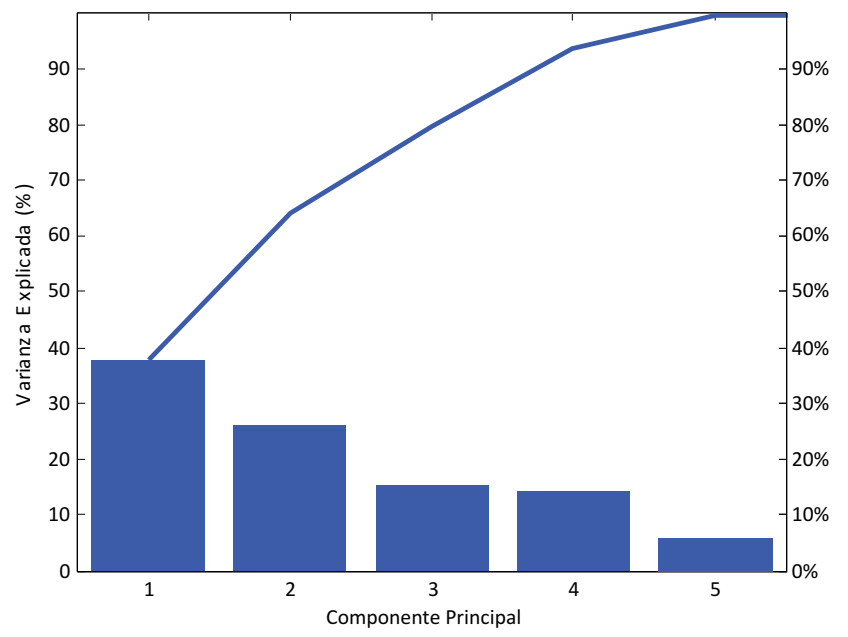

Figura 11. Varianza explicada y varianza acumulada para los cinco componentes principales de los caracteres morfométricos de semillas de G. americana de las seis procedencias. 
Los resultados morfométricos obtenidos se asemejan a lo reportado en la literatura: semillas planas con 1$1,20 \mathrm{~cm}$ de longitud (2).

En esta investigación, las semillas presentaron formas irregulares con cierta tendencia a ser elipsoides y achatadas; aunque algunos autores mencionan que la mayoría de semillas presentan forma deltoide (13). El tamaño fue superior a lo reportado en la literatura (4).

En el caso de la variable peso de las semillas; se encontraron diferencias con lo reportado (4-9), siendo mayores los pesos obtenidos en la presente investigación.

El tamaño, peso y forma de las semillas puede estar influenciado por el tamaño de los frutos del que proceden, que en general es mayor del reportado en la literatura para los seis sitios de colecta.

La forma de la semilla está determinada genéticamente y es moldeada por el espacio para su crecimiento dentro del fruto e influenciada por el tipo de óvulo del cual se ha originado; tiene función en la orientación de la semilla en la caída para su germinación y en la dispersión de la semilla anemócora o hidrócora (11).

Algunas características de las semillas como el grosor y ancho de la semilla están influenciados por factores ambientales tales como humedad, temperatura y $\mathrm{pH}$. Estos caracteres son de gran importancia, ya que el tamaño de la semilla influye en el vigor de las plántulas, el cual juega un papel en su sobrevivencia (12).

Así mismo, el tamaño y peso de la semilla están determinados genéticamente y son afectados por factores ambientales durante el desarrollo de la semilla (11).

La variación intraespecífica en tamaño y peso es influenciada por el empaquetamiento dentro del fruto. El peso de las semillas es afectado por el tamaño, estructura y contenido y proporciones celulares.

En relación a lo anterior, el fruto tipo baya de G. americana produce semillas que van desde 50 hasta más de 300 semillas cada uno, lo cual influye notablemente en los caracteres morfológicos de la semilla. La variación en peso es afectada por la exposición al aire, ya que las semillas están envueltas en fibras jugosas del mesocarpo y al ser retiradas éstas, empiezan a perder humedad rápidamente por su alta tasa metabólica y adaptación para una rápida germinación en condiciones de alta humedad en el suelo, como lo confirma un estudio sobre almacenamiento de estas semillas realizado en 2010 (13).

La morfometría de las semillas de G. americana difiere según el sitio de procedencia. Esto puede deberse a la acción de factores como el tipo de suelo, la cantidad de radiación solar o lluvia y la edad del árbol que influye de alguna forma en las características de la semilla madura.

De acuerdo con estos resultados es probable que las semillas tengan mayor influencia del ambiente en las características de volumen, peso, longitud, ancho y grosor de las semillas. El tamaño de la semilla es una característica variable en muchas poblaciones de plantas; éste a menudo influye en la sobrevivencia, crecimiento y reproducción.

Es posible que la poca variación genética para características correlacionadas con éxito reproductivo (debido a un período largo de selección y eliminación de alelos con efectos deteriorantes en el éxito reproductivo) tales como el tamaño de la semilla, explique el origen de la variación principalmente de recursos ambientales (12).

La alta variabilidad intrapoblacional en los árboles forestales, puede constituir una respuesta adaptativa a la heterogeneidad espacial y temporal encontrada en las poblaciones. Además, la variación morfológica de una especie vegetal se encuentra íntimamente ligada a las condiciones medioambientales que prevalecen en el ciclo de vida de la misma, de modo que la estrecha relación planta-medio ambiente marca la propiedad de la especie vegetal de poseer la suficiente flexibilidad de desarrollo o normas de reacción amplias para existir en las condiciones donde se ubica (6).

Las diferencias encontradas en los caracteres evaluados, podrían ser benéficas para mantener la diversidad genética en la población. Cabe en particular destacar que es muy probable que las especies con semillas de mayor peso y tamaño tengan mayor oportunidad de sobrevivir las primeras fases de su ciclo de vida.

Esto se confirma con la correlación detectada por diversos autores (6) entre peso de la semilla y capacidad de germinación, lo cual puede tener implicaciones en la producción de vivero. 


\section{CONCLUSIONES}

- Los frutos y semillas de G. americana presentan frutos y semillas con medidas superiores a lo reportado en la literatura.

- Las semillas de G. americana presentan en su mayoría forma irregular y la mayor variación morfométrica corresponde a características como volumen, peso y largo, independientemente de la procedencia de las mismas.

- Factores genéticos y ambientales pueden estar afectando el tamaño, peso y forma de la semilla, las cuales influencian la sobrevivencia, crecimiento y reproducción.

\section{AGRADECIMIENTOS}

Los autores del proyecto agradecen a:

- Centro del Estudios e Investigaciones en Biodiversidad y Biotecnología de la Universidad del Quindío (CIBUQ). Instalaciones, equipos, insumos y material de campo.

- Reserva Natural La montaña del Ocaso. Apoyo logístico.

- Jamid Escobar y Leider Suárez. Guardaselvas del Ocaso.

- M. Sc. Rocío Stella Suárez R, y Asp. M. Sc. Lina Marcela Arbeláez Arias. Por su asesoría durante el desarrollo de este proyecto.

\section{BIBLIOGRAFÍA}

1. Mendoza, H., Ramírez, B. \& L.C. Jiménez. Rubiaceae de Colombia-Guía ilustrada de géneros. Instituto de Investigación de Recursos Biológicos Alexander Von Humboldt. Bogotá. Colombia. 2004. 351 p.

2. Jøker, D., DFSC, Nassif Salomão, A., Vásquez, C. y W. Vásquez, CATIE. Genipa Americana L. 2003. Seed Leaflet. No.67, 2 p.

3. Andrade, A.C.S., Souza, A.F, Ramos, F.N., Pereira, T.S. \& A.P.M. Cruz. Germinação de sementes de Jenipapo: Temperatura, substrato e morfologia do desenvolvimento pós-seminal. Pesq. agropec. bras., Brasília, v.35, n.3, mar, 2000. p.609-615.

4. Silva, D.B., Salomão, A.N., Caravalho. P.C.L. \& M.M.V.S. Wetzel. Jenipapo. En: Vieira, R. F. (ed.) Frutas nativas da região Centro-Oeste do Brasil. Brasília: Embrapa Recursos Genéticos e Biotecnología, 2006. p. 304-322.

5. Oficina para la Coordinación de Asuntos Humanitarios / Naciones Unidas Colombia. OCHA. [en línea] Página Web versión HTML. Bogotá, Colombia: (s.e.), 2006. [citado 12 de Agosto de 2009]. Disponible en Internet: <http://www.colombiassh.org/site/spip.php?article27>.

6. Iglesias, L., Mora, I. y J.L. Casas. Morfometría, Viabilidad y variabilidad de las semillas de la Población de Pinus hartwegii del cofre de Perote, veracruz, México. Cuadernos de Biodiversidad Número 19. Publicación cuatrimestral del Centro Iberoamericano de la Biodiversidad 2005.

7. Renhe, I.R.T. Extraçao e estabilidade do corante azul de jenipapo (Genipa americana L). Dissertação (mestrado). Programa de Pós-Graduação em Ciência e Tecnologia de Alimentos. Universidade Federal de Viçosa. Minas Gerais. Brasil. 2008.49p.

8. Salomão, A.N. \& L. S. Padilha. Avaliação preliminar da germinabilidade e da micoflora associada às sementes de Genipa americana em diferentes estágios de maturação. Brasília: Embrapa Recursos Genéticos e Biotecnologia, 2006 (Série Embrapa -Circular Técnica 50). 9 p.

9. Salomão, A.N. Desiccation, storage and germination of Genipa Americana seeds. En: Sacandé, M., D. Joker, M.E. Dulloo \& K.A. Thomsen. Comparative Storage Biology of Tropical Tree Seeds. International Plant Genetic Resources Institute, Rome, Italy. 2004. 363 p.

10. Crestana, C.S.M., Batista, E.A. \& G. Mariano. Fenologia da frutificação de Genipa americana L (Rubiaceae) em mata ciliar do Rio Moji Guaçu, SP. IPEF, n.45, jan./dez.1992. p.31-34. 
11. Werker, E. 1997. Seed anatomy. Encyclopedia of plant anatomy. Tomo X, 3. Gebrüder Borntraeger, Berlín.

12. Iglesias, L., y Y. Tivo, F. Caracterización morfométrica de la población de Pinus hartwegii Lindl. del Cofre de Perote, Veracruz, México. Ra Ximhai, Vol.2. Universidad Autónoma Indígena de México Mochicahui, El Fuerte, Sinaloa. 2006, pp. 449-468.

13. Cherobini, E.A.I. Avaliação da qualidade de sementes e mudas de espécies florestais nativas. Dissertação (mestrado). Programa de Pós-Graduação em Engenharia Florestal, Área de Concentração Silvicultura, da Universidade Federal de Santa Maria. R. S. Brasil. 2006. 114 p.

14. Nascimento, W.M.O. \& C. F. Damião-Filho. Caracterização morfológica de sementes e plántulas de Jenipapeiro (Genipa americana L. - Rubiaceae). Revista Brasileira de Sementes, vol. 20, no 1. 1998. p.143-147.

15. Schmidt L. Guide to Handling of Tropical and Subtropical Forest Seed. Danida Forest Seed Centre. Humleback (Dinamarca). 2000.511 p. 